\title{
Funktionelle Anatomie des proximalen Femur
}

\author{
Rosemarie Fröber
}

\section{Zusammenfassung}

Das proximale Femurende ist physiologischerweise hohen Druckund Biegebeanspruchungen ausgesetzt und unterliegt infolge funktioneller Anpassung einem ständigen Knochenumbau. Da Schenkelkopf und -hals in besonderer Weise von traumatischen Ereignissen und degenerativen Veränderungen betroffen sind, sollen ausgewählte anatomische Details dieses Femurabschnittes einer genaueren Betrachtung unterzogen werden.

\section{Einleitung}

Der Oberschenkelknochen, das Femur, ist der größte Röhrenknochen des menschlichen Körpers. Er hat wesentlichen Anteil an der Körpergröße und besteht als typischer Röhrenknochen aus einer Diaphyse und zwei Epiphysen. Das Caput ossis femoris, das im Hüftgelenk, Articulatio coxae, mit der Hüftpfanne, Acetabulum, in gelenkiger Verbindung steht, gleicht geometrisch gesehen zwei Dritteln einer Kugel. An seiner Knorpel-Knochengrenze, die beim wachsenden Skelett der proximalen Epiphysenfuge entspricht, geht der Schenkelkopf in den Schenkelhals über. Zur Diaphyse gehören Collum und Corpus ossis femoris. An der Grenze zwischen Schenkelhals und -schaft befinden sich die beiden Rollhügel, Trochanter major et minor, die dem Ansatz kräftiger Muskeln dienen und zu den tastbaren, diagnostisch wichtigen Landmarken des Hüftgelenkes gehören. Der von Schenkel-

OP-JOURNAL 2002; 17: 86-90

(c) Georg Thieme Verlag Stuttgart . New York
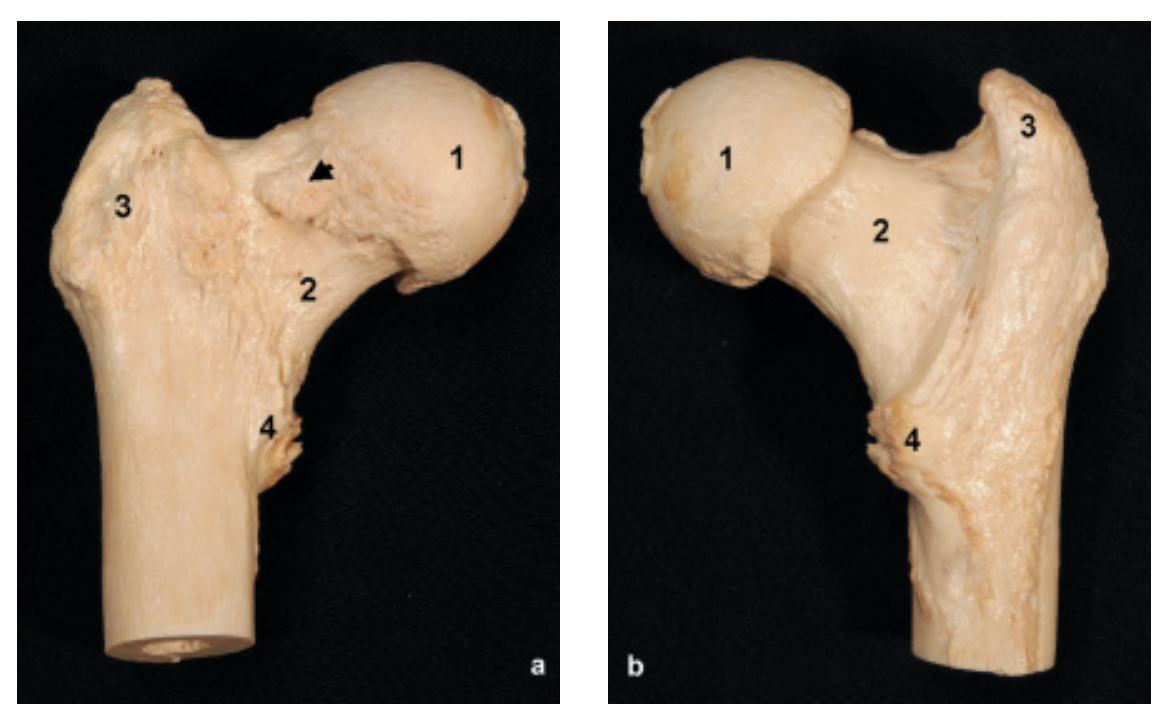

Abb.1 Proximales Femurende einer 76-jährigen Frau mit auffälligen degenerativen Veränderungen (Pfeil). (a) Ansicht von vorn, (b) Ansicht von hinten. 1 Schenkelkopf, 2 Schenkelhals, 3 großer und 4 kleiner Rollhügel.

hals und -schaft gebildete Collum-CorpusWinkel (fälschlicherweise häufig als Collum-Diaphysen-Winkel bezeichnet) ist für die Stabilität des proximalen Femur (Abb. 1 a u. b) von entscheidender Bedeutung. Die Häufigkeit von Schenkelhalsfrakturen im Alter ist neben dem Abbau und Elastizitätsverlust des Knochengewebes durch den sich verkleinernden Collum-Corpus-Winkel bedingt. Die Winkeleinstellung (125 $5^{\circ}$ Normwert des Erwachsenen) beruht auf einer funktionellen Anpassung des Oberschenkelknochens an seine tägliche Beanspruchung.

\section{Architektur des proximalen Femur}

Die Belastung des Femur erfolgt durch Körpergewicht sowie durch Muskelund Bandkräfte. An seiner Belastung ist daher ein Kräftepaar mit entgegengesetzten Wirkungsrichtungen beteiligt, so dass letztlich die Resultierende aus beiden Kräften Femur und zugehörige Gelenke belastet. Da die Resultierende diaphysenwärts exzentrisch verläuft, kommt es zu
Druck- und Biegebeanspruchungen. Knochengewebe passt sich nach Pauwels der einwirkenden Belastung in zweierlei Weise an. Einerseits werden Menge und Verteilung von Kortikalis und Spongiosa des Knochens entsprechend reguliert; andererseits erfährt auch die trajektorielle Ausrichtung der Spongiosaarchitektur eine funktionelle Anpassung. Knochen ist also nach ökonomischem Bauprinzip konstruiert, wobei mit einem Minimum an Material ein Maximum an Festigkeit erzielt wird.

Bei normalem Schenkelhalswinkel wird das proximale Femur sowohl auf Druck als auch Biegung beansprucht. Die Spongiosatrabekel dieser Region, die man sowohl im Sägeschnitt (Abb. 2a) des Knochens als auch im Röntgenbild gut erkennen kann, richten sich in der Weise aus, dass sie ausschließlich auf Druck oder Zug beansprucht werden und keinerlei Biegebeanspruchung mehr erfahren (Transformationsgesetz nach Wolff; biomechanisch nachgewiesen von Pauwels). 

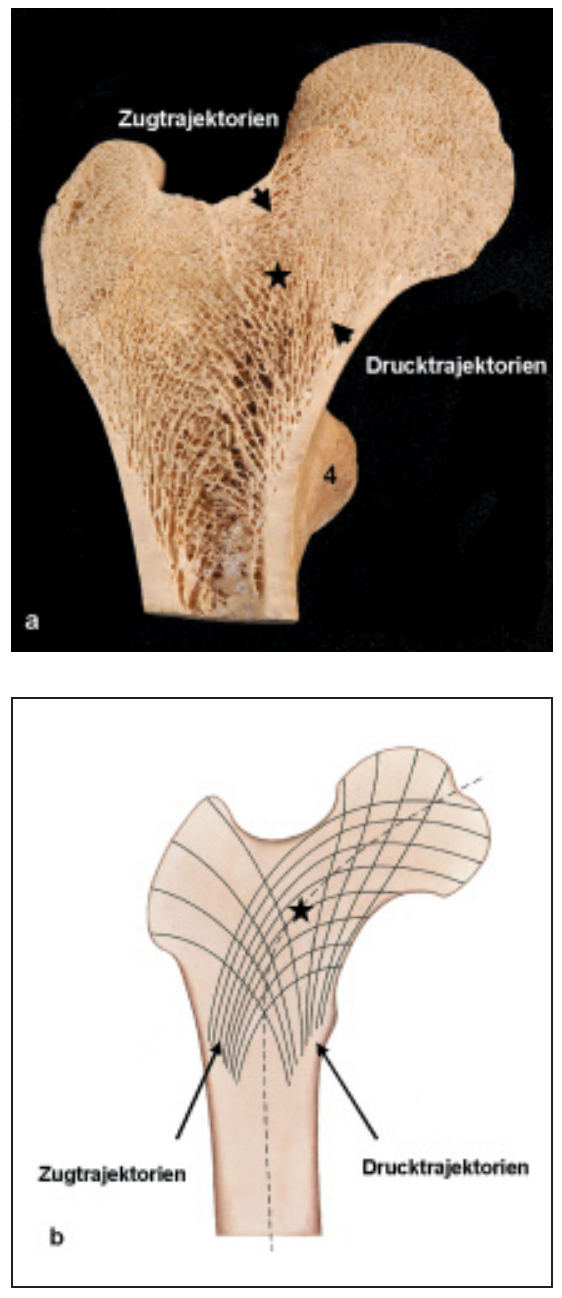

Abb. 2 Trajektorienverlauf im proximalen Femur bei normalem Schenkelhalswinkel und normaler Belastung. Zwischen Druck- und Zugtrajektorien liegt das Wardsche Dreieck (Stern). (a) vertikaler Sägeschnitt und (b) Schema in der Ansicht von vorn.

Die Drucktrabekel (Abb. 2 b) entspringen im kranialen Bereich des Femurkopfes und strahlen kaudal in die Kortikalis auf der medialen Seite des Schenkelhalses ein (Adamscher Bogen). Sie werden von Zugtrabekeln rechtwinklig gekreuzt, die aus dem unteren Bereich des Femurkopfes kommen und bogenförmig zum lateralen Abschnitt des Schenkelhalses verlaufen, bevor sie weiter kaudal in die laterale Kortikalis des Schaftes einmünden. Die Zugtrabekel werden nochmals von Trajektorien, die zwischen beiden Rollhügeln verlaufen, gekreuzt. Ein im Inneren des Schenkelhalses gelegenes, auch im Röntgenbild zwischen den drei Trabekelsystemen erkennbares Feld geringerer Knochendichte wird als Wardsches Dreieck bezeichnet. Die Trajektorien von Schenkelkopf und -hals zeigen genau den Verlauf von Druck- (Kompression)

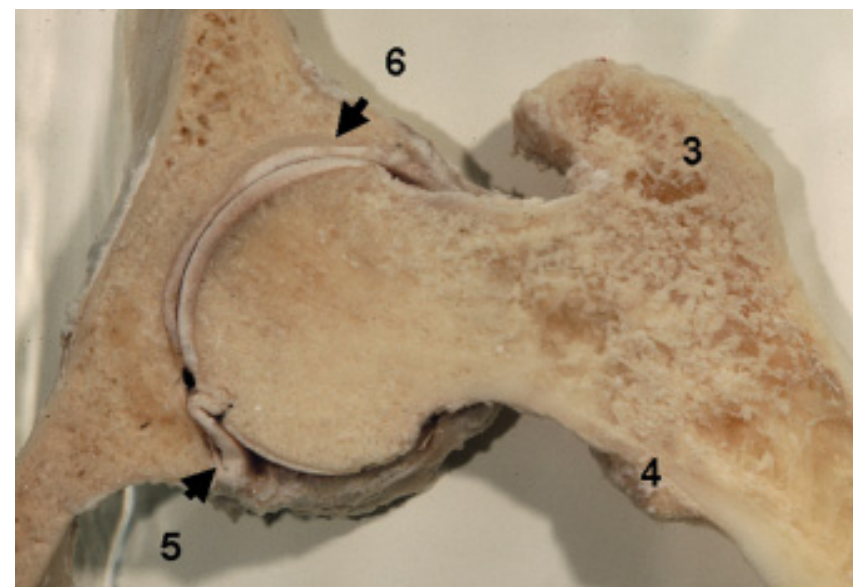

Abb. 3 Hüftgelenk. Vertikalschnitt in der Ebene des Schenkelhalses. Das Lig. capitis femoris (5) verbindet den Schenkelkopf mit der basal gelegenen Inzisur der Pfanne. Die artikulierenden Flächen von Pfannendach und Schenkelkopf sind von dickem Knorpel (6) bedeckt.

oder Zugspannungen (Dehnung) an. Biegung wird durch funktionelle Anpassung der Spongiosaarchitektur völlig ausgeschaltet.

Bei der sich im Alter einstellenden Coxa vara (verkleinerter Schenkelhalswinkel) nimmt die Biegebeanspruchung des proximalen Femurs, und damit die Beanspruchung insgesamt erheblich zu. Den vermehrten Zugspannungen entsprechend verstärken sich die an der lateralen Seite des Schenkelhalses verlaufenden Zugtrabekel. Die Frakturgefahr ist erhöht.

Durch Zuggurtung (Muskeln, Faszien und Bänder) werden Biegebeanspruchungen stark herabgesetzt. Am menschlichen Femur übernehmen die das Hüftgelenk umgebenden Muskeln (M. gluteus maximus und ischiocrurale Muskulatur, M. rectus femoris und Tractus iliotibialis) und Bänder (Ligg. iliofemorale, ischiofemorale, und pubofemorale) die Funktion, die Biegebeanspruchung des proximalen Femurabschnittes in physiologischen Bereichen zu halten.

Ist die Zuggurtung des Femurs infolge von nachlassender Muskelkraft, Bandschäden oder Änderungen der Knochengestalt (postoperativ, posttraumatisch oder infolge von Erkrankungen des Knochens) nicht oder nur vermindert wirksam, so kann es schon bei leichten Beanspruchungen zu proximalen Femurfrakturen kommen.

\section{Labrokapsulärer Komplex des Hüftgelenkes}

Da die Gelenkkörper des Hüftgelenkes, Articulatio coxae, nicht absolut kongruent sind, führen bereits sehr geringe Verschiebungen zu erheblichen Deformierungen des Acetabulum. Die Pfanne ist daher zu ihrer eigenen Stabilisierung mit massiven, zugfesten Einrichtungen ausgerüstet. Dazu gehört ein C-förmiger Gelenkknorpel, die Facies lunata, der die Pfanne unvollständig ausfüllt (Abb. 3). Er nimmt sehr hohe Zugkräfte auf, was zu erheblichen Dehnungsverformungen führt. Diese werden nicht nur auf die knöcherne Pfanne übertragen, sondern auch auf das Lig. transversum acetabuli, das die beiden freien Enden des Pfannenknorpels miteinander verbindet und mit einer echten Gelenklippe, Labrum acetabuli, fest verwachsen ist. Der nicht von Knorpel überzogene Pfannenabschnitt, Fossa (Pulvinar) acetabuli, ist mit einem von Synovia bedeckten Bindegewebs- und Fettpolster ausgestattet, dem eine Stoßdämpferfunktion zukommt. Er beherbergt das gefäßführende Lig. capitis femoris (Lig. teres), ein die Gelenkkörper verbindendes intraartikuläres Band (Abb. 4a u. b).

Das Labrum präsentiert sich als ringförmige Struktur in sich verdrehter kollagener Faserbündel, die streng zirkulär ausgerichtet sind und in den knöchernen Rand der Gelenkpfanne, Limbus acetabu$l i$, einstrahlen. Die Gelenklippe sitzt dem Limbus breitbasig auf, setzt sich aber von der Knorpelabdeckung der Pfanne bis auf eine kleine kraniodorsale Zone durch einen scharfen Spalt ab. Der äußere faserhaltige Anteil der Kapsel nimmt am knöchernen Rand der Pfanne seinen Ursprung. Dadurch springt die Gelenklippe frei in den Gelenkinnenraum vor (Abb. 5). Das Labrum, dessen Rolle sich von der physiologischen, lastabhängigen Inkongruenz der Gelenkkörper ableitet, bildet eine feste Zuggurtung des Pfannenrandes. Es stellt sich als derber Bindegewebskörper dar, der an seiner Außenseite von der Innenschicht der Gelenkkapsel, der Synovialmembran, abgedeckt wird. 

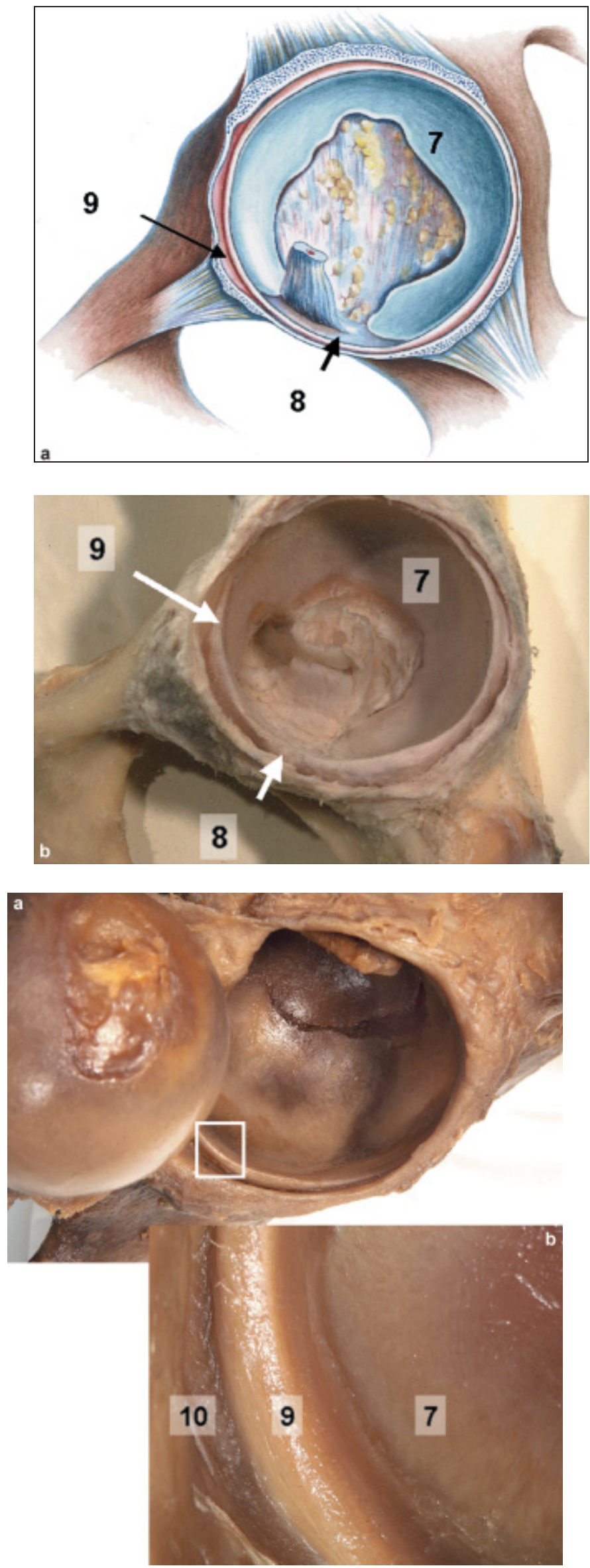

Abb. 4 Linkes Acetabulum mit halbmondförmigem Gelenkknorpel [7], Lig. transversum acetabuli [8] und in den Gelenkraum vorspringender Gelenklippe [9], (a) schematisch und (b) in situ.

Abb. 5 Linkes Hüftgelenk in situ eröffnet. Blickrichtung zum Pfannendach mit Gelenkknorpel [7], Gelenklippe [9] und Synovialmembran [10]. (a) Übersicht und (b) Ausschnittsvergrößerung.
Pfannenknorpel, Querband und Gelenklippe dienen der zirkulären Integrität der Gelenkpfanne. Sie reduzieren letztlich die Belastung der knöchernen Pfanne

Die Gelenkkapsel selbst ist dick und fest. Am Femur inseriert ihre Außenschicht, die durch massive Gelenkbänder (Ligg. iliofemorale, ischiofemorale und pubofemorale) verstärkte Faserkapsel, ventralseitig an einer die beiden Rollhügel verbindenden Knochenlinie; rückseitig ist sie zwischen mittlerem und lateralem Drittel des Schenkelhalses verankert. Der innere, synoviale Kapselanteil lässt das gesamte laterale Drittel des Halses frei. Er überzieht die mediale $2 / 3$-Region und erstreckt sich bis zur Knorpel-Knochengrenze des Schenkelkopfes (Abb. 6).

Eine zwischen Synovialmembran und Knochen befindliche dünne Bindegewebsschicht führt nutritive Gefäße zum Schenkelkopf, die z.T. deutliche Synovialmembranfalten aufwerfen.

Die Lücken zwischen den massiven Verstärkungsbändern sind Schwachstellen in Bezug auf die Stabilität des Hüftgelenkes. Hier kann bei traumatischer Hüftgelenkluxation der Femurkopf nach außen treten.

\section{Vaskularisation und Innervation}

Der distale Gelenkkörper des Hüftgelenkes weist hinsichtlich seiner aus unterschiedlichen Quellen stammenden Vaskularisation außergewöhnliche anatomische Verhältnisse auf. Diese besitzen eine hohe klinische Relevanz:

Äste der A. nutricia proximalis des Femurschaftes beteiligen sich lediglich im Bereich der Innenschicht der Kortikalis an der Versorgung des Schenkelhalses, wobei diese bis zur Epiphysenfuge des Schenkelkopfes reicht.

Die Außenschicht der Schenkelhals-Kortikalis erhält ihre Blutversorgung aus einem an der Basis des Schenkelhalses, außerhalb der Gelenkkapsel verlaufenden Arterienring. Dieser wird von der kürzeren A. circumflexa femoris lateralis und der längeren $A$. circumflexa femoralis medialis gespeist (Abb. 7a) und ist in 50\% der Fälle geschlossen. In der Regel entspringen beide Arterien getrennt oder über einen kurzen gemeinsamen Stamm aus der $A$. profunda femoris; seltener nimmt eine der Arterien ihren Ursprung direkt aus $\operatorname{der} A$. femoralis. 

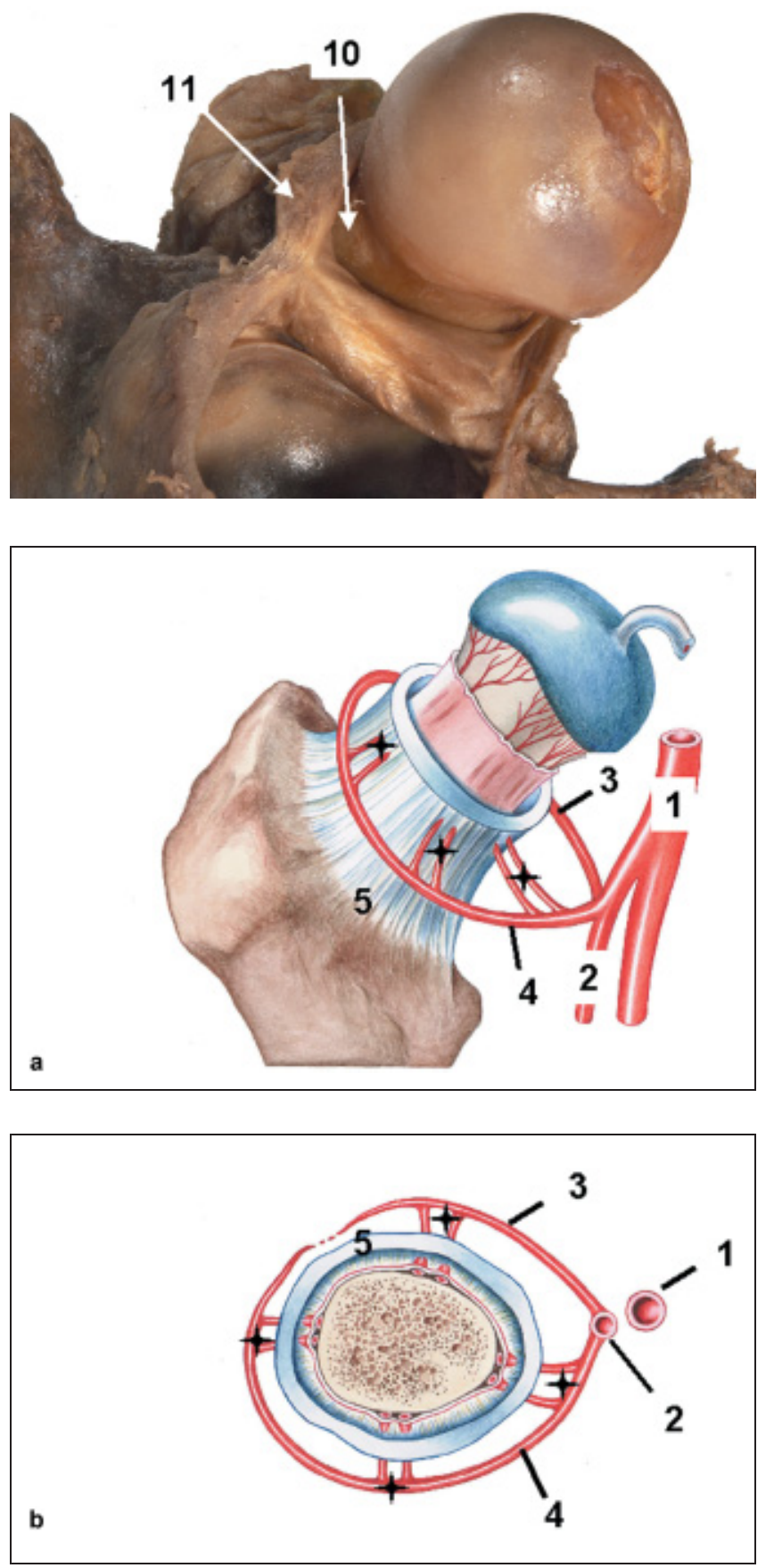

Die maßgeblich an der Blutversorgung von Schenkelhals und -kopf beteiligte A. profunda femoris ist bei der operativen Versorgung von proximalen Femurfrakturen gefährdet.

Vom Gefäßring ausgehend erfolgt die Hauptversorgung des proximalen Femurs über eine Vielzahl von Verzweigungen, die über zahlreiche Foramina nutricia den Knochen erreichen. Von besonderer Bedeutung sind vier Gruppen aszendie-
Abb. 6 Linkes Hüftgelenk in situ eröffnet. Blickrichtung zur

Synovialmembranabdeckung des Schenkelhalses [10] und zur durch Bänder verstärkten Gelenkkapsel [11].

Abb. 7 Arterielle Versorgung des proximalen Femurs (nach Putz 99).

(a) Ansicht von vorn,

(b) Querschnitt.

1 A. femoralis,

2 A. profunda femoris, 3 A. circumflexa femoris medialis, 4 A. circumflexa femoris lateralis, 5 Gelenkkapsel und aszendierende Gefäße (Stern).

render Arterien, die jeweils aus zwei bis drei Gefäßen bestehen und die Gelenkkapsel durchbohren, um zur Gelenkinnenhaut zu gelangen (Abb. 7b). Aus der A. circumflexa femoris lateralis entspringt eine vordere, mediale und laterale Astgruppe; eine hintere entstammt der $A$. circumflexa femoris medialis. Subsynovial verlaufen die Äste dem knöchernen Schenkelhals unmittelbar anliegend bis zur Knorpel-Knochen-Grenze. Hier verzweigen sie sich in zwei bis drei weitere Ästchen und treten von distal kommend
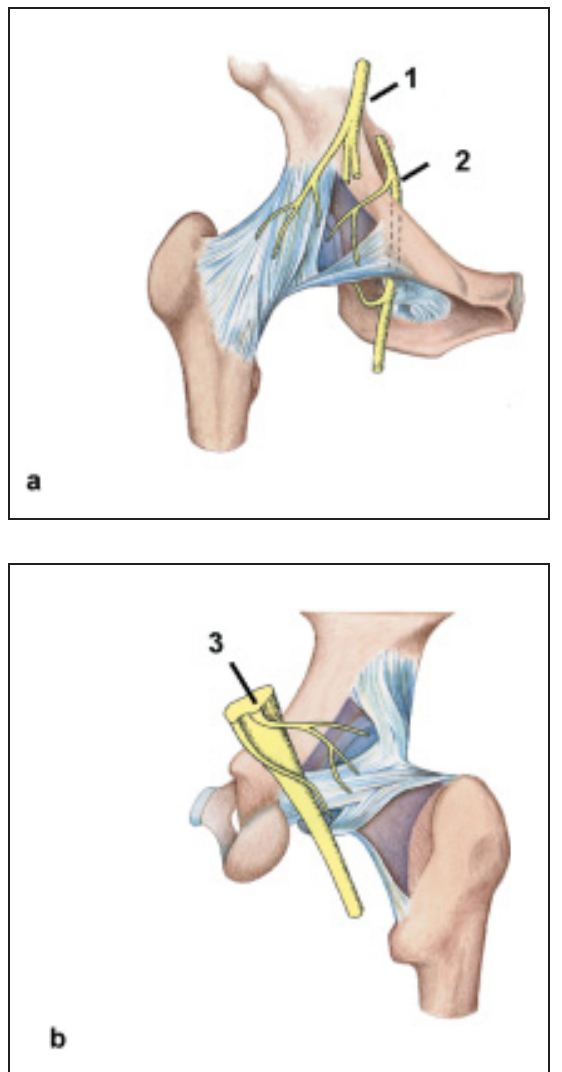

Abb. 8 Innervation des Hüftgelenkes (nach Putz 98). (a) Ansicht von ventral und (b) Ansicht von dorsal. $1 \mathrm{~N}$. femoralis, $2 \mathrm{~N}$. obturatorius, 3 Plexus sacralis mit N. glutaeus inferior, $\mathrm{N}$. ischiadicus und R. m. quadrati femoris.

über Foramina nutricia in den Knochen des überknorpelten Schenkelkopfes ein, um diesen zu versorgen.

Bei (insbesondere medialen) Schenkelhalsfrakturen werden die subsynovial verlaufenden nutritiven Gefäße des Schenkelkopfes in Mitleidenschaft gezogen.

Im Wachstumsalter besitzt das Gebiet der Kopfepiphyse jenseits der Wachstumsfuge eine völlig eigenständige Vaskularisation. Diese wird über die aus der A. obturatoria stammende Pfannenarterie, welche via Ligamentum capitis femoris den Schenkelkopf erreicht, realisiert. Bereits beim älteren Kind überspringen zahlreiche, von distal kommende aszendierende Gefäße die Wachstumsfuge (vor allem an deren lateralem Umfang) und übernehmen die Blutversorgung des Schenkelkopfes. Die im Kopfband verlaufende Pfannenarterie spielt daher für die spätere Ernährung des Schenkelkopfes keine bedeutende Rolle mehr. 
Zerreißen die auf dem Schenkelhals verlaufenden nutritiven Gefäße, so kann es zur Nekrose des Knochengewebes im Femurkopf kommen.

Die Innervation des Hüftgelenkes erfolgt durch zahlreiche Verzweigungen der dem Hüftgelenk benachbarten Nerven. Diese treten von ventrolateral und -medial sowie von dorsal in den fibrösen Anteil der Gelenkkapsel ein und bilden hier freie Nervenendigungen aus oder nehmen mit Rezeptororganen Kontakt auf.

Auf der Vorderseite handelt es sich um Äste des N. femoralis und des N. obturatorius, während sich auf der Rückseite Endäste des $N$. glutaeus inferior sowie Muskeläste (für den M. quadratus femoris) aus dem Plexus sacralis an der Innervation der Gelenkkapsel beteiligen (Abb. 8). Die Nerven dienen der Schmerzperzeption (über freie Nervenendigungen) sowie der Mechanorezeption (bei- spielsweise über Golgi-Mazzoni-, VaterPacini- und Ruffini-Körperchen). Letztere verteilen sich als Registratoren der Tiefensensibilität zur Wahrnehmung von Spannungen bzw. Spannungsänderungen über die gesamte Faserkapsel des Hüftgelenkes.

Die nervale Versorgung der labrokapsulären Strukturen dient nicht nur als Warnsystem des Hüftgelenkes (Schmerzrezeption), sondern ist auch in die sensomotorische Kontrolle von Gelenkstellungen und -bewegungen (Mechanorezeption) integriert.

\section{Danksagung}

An dieser Stelle sei Herrn Jens Geiling, Wissenschaftsgraphiker am Institut für Anatomie I, und Herrn Michael Szabo, Fotograf am klinischen Medienzentrum der Friedrich-Schiller-Universität Jena, für die wertvolle Mitarbeit gedankt.

\section{Literatur}

1 Lanz-Wachsmuth. Praktische Anatomie. Band I, Teil 4. Berlin: Springer 1938

2 Pauwels F. Über die Verteilung der Spongiosadichte im coxalen Femurende und ihre Bedeutung für die Lehre vom funktionellen Bau des Knochens. Morph. Jb. 1955; 95 $35-54$

3 Putz R, Schrank C. Anatomie des labrokapsulären Komplexes. Orthopäde 1998; 27: 675 680

4 Putz R, Kaiser E. Vaskularisation des Schenkelhalses. Deutsche Gesellschaft für Chirurgie. Kongressband 1999

5 Rauber/Kopsch. Anatomie des Menschen. Band I. Hrsg. B. Tillmann und G. Töndury. Stuttgart, New York: Georg Thieme 1987

\section{Dr. med. Rosemarie Fröber}

Prosektor am Institut für Anatomie I

Medizinische Fakultät

Friedrich-Schiller-Universität Jena

D-07740 Jena 